\title{
LOS FONDOS DE PENSIONES Y SU DESEMPEÑO DURANTE LA CRISIS 2007 - 2012
}

\author{
PENSION FUNDS AND ITS PERFORMANCE DURING THE CRISIS 2007 - 2012 \\ Adrián Alejandro Flores Konja * \\ Docente Principal de la Facultad de Ciencias Contables \\ David Juan Sanchez Cruz ** \\ Docente Contratado de la Facultad de Ciencias Contables \\ Universidad Nacional Mayor de San Marcos-UNMSM / Lima-Perú \\ [Recepción: Febrero 2016/ Conformidad: Mayo 2016]
}

\section{RESUMEN}

El presente artículo analiza el desempeño de los fondos de pensiones administrados por las AFPs que operan en el Perú para los años entre el 2007 y el 2012, años caracterizados por una crisis que generó preocupación en un tema importante para los peruanos en edad de jubilación o próximos a jubilarse: las pensiones. Tal preocupación se justifica cuando se tiene en cuenta que los ahorros depositados en fondos de pensiones están invertidos en mercados financieros afectados por la crisis generada en el mercado norteamericano. Este trabajo evalúa los riesgos generados por la crisis financiera para los fondos de pensiones. Las evidencias obtenidas muestran que los administradores de portafolio de las AFPs asumen riesgos incluso por encima de los riesgos asumidos en el Mercado Bursátil Peruano. Por otro lado, la evaluación de desempeño de gestión para las AFPs muestra la ausencia de un comportamiento competitivo.

\section{Palabras Clave:}

Crisis hipotecaria; sistema de pensiones; medidas de desempeño.

\begin{abstract}
This article analyzes the performance of pension funds managed by the AFPs operating in Peru, in the years between 2007 and 2012, years characterized by a crisis that raised concern over an important issue for Peruvians in retirement age or close to retirement: the pensions. Such concern is justified when you consider that the savings deposited in pension funds are invested in financial markets affected by the crisis generated in the US market. This paper assesses the risks posed by the financial crisis for pension funds. Evidence obtained show that the portfolio managers of the AFPs take risks even over to those assumed in the Peruvian Stock Market. On the other hand, the performance evaluation management for AFPs shows the absence of a competitive behavior.
\end{abstract}

\section{Keywords:}

Mortgage crisis; pension system; performance measure.

\footnotetext{
* Doctor en Ciencias Contables -UNMSM. Email: afloreskonja@yahoo.es

** Magíster en Economía-UNMSM. Email: davidsanch_c@hotmail.com
} 


\section{INTRODUCCIÓN}

A mediados del 2007 el banco Bear Stearns comunica a sus clientes que es poco probable que recuperen su dinero invertido en sus dos hedge funds en dificultades. El banco de inversión norteamericano achaca la situación a la pérdida de valor de las obligaciones cuyo colateral eran las hipotecas de baja calidad o subprime. El 15 de Septiembre del 2008, Lehman Brothers se declara insolvente, asimismo, Merrill Lynch es vendido al Bank of America y AIG solicita con urgencia un multimillonario crédito de la FED. Estos hechos agudizan la crisis, que como una pandemia, se expande por el mundo.

El Perú no fue ajeno a estos efectos negativos, pues los precios de los títulos se derrumbaron, lo que desvalorizó los portafolios de personas e instituciones que colocaron sus excedentes en la Bolsa de Valores de Lima. Las AFPs con cuantiosas inversiones en acciones e instrumentos de deuda solo atinaron a comunicar a sus clientes que la crisis financiera norteamericana fue la causa de las pérdidas en sus fondos de pensiones y que en el largo plazo todo volvería a su nivel. ¿Fueron estas explicaciones suficientes? ¿Las AFPs son conscientes de la preocupación de los peruanos respecto a sus futuras pensiones? ¿Las AFPs están incentivadas para realizar una gestión apropiada del riesgo y la rentabilidad? ¿Los retornos obtenidos son mejores a los que ofrecen otras instituciones?
En fin, existen muchos cuestionamientos que merecen respuestas transparentes y profesionales, $y$ que demandan tiempo y recursos para su investigación. Por nuestra parte, analizaremos la gestión de los fondos de pensiones a la luz de lo que plantea la teoría y la práctica financiera. Es la oportunidad de evaluar su desempeño durante un periodo de crisis que sin duda volverá a repetirse.

\section{PROBLEMA}

La crisis hipotecaria generada en el mercado financiero norteamericano ha sido una de las pruebas más difíciles para el Sistema Privado de Pensiones que administra los fondos de pensiones de millones de peruanos. Respecto a esta situación, resulta válido efectuar los siguientes cuestionamientos:

1. ¿ Se gestionó eficientemente el riesgo de mercado? 2. ¿Los rendimientos obtenidos tuvieron relación con los riesgos asumidos?

\section{LA CRISIS FINANCIERA 2007 - 2011}

La crisis de las hipotecas subprime estalló a comienzos de 2007 en los EUA y luego se propagó por toda la economía mundial ${ }^{1}$. Como apreciamos en la figura $\mathrm{N}^{\circ} 01$ el índice Dow Jones llegó a su máximo en el tercer trimestre de 2007 para luego iniciar la caída que se agudizó el 15 de septiembre de 2008 con el anuncio de banca rota de Lehman Brothers.

\section{Figura No 01:}

Evolución del Dow Jones (Enero de 2006 a noviembre de 2008)

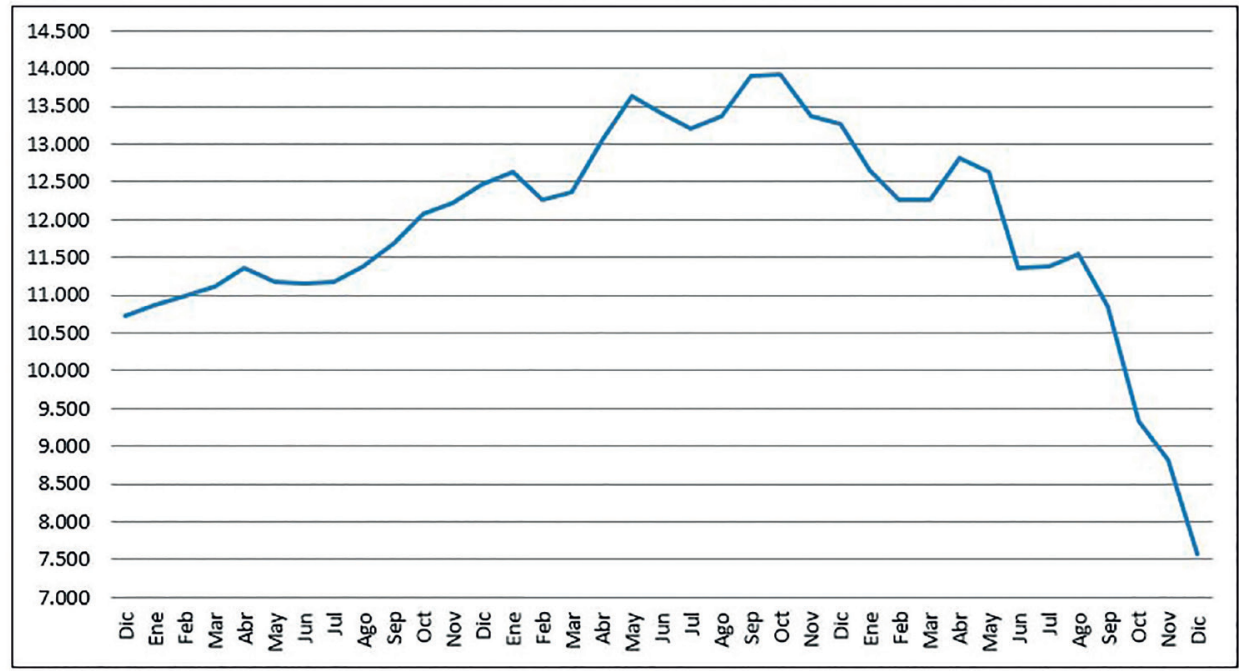

Fuente: BBC News UK.

1 BBC News UK. 2009. Financial Crisis: How it happened.

100/ QVIPURAMAYOC | Vol. 24(45 A) 2016 


\section{LA CRISIS Y LOS FONDOS DE PENSIONES EN EL PERÚ}

Como apreciamos en la figura $\mathrm{N}^{\circ} 02$ los fondos de pensiones en poder de las AFPs han experimentado un crecimiento continuo, pero sufre una fuerte e inusual caída en 2008, de la que se recupera y vuelve a caer en $2011^{2}$. Es importante indicar que en 2008 los fondos de pensiones ya representaban el $18 \%$ del $\mathrm{PBI}$, es decir, una cifra muy considerable.

Para 2009, la crisis financiera mundial afectó negativamente el crecimiento del PBI peruano, que apenas alcanzó 0.9\%. En el 2010, la economía peruana creció $8.8 \%$ impulsada por el dinamismo de la demanda interna.

Figura No 02:

Evolución del fondo de pensiones vs PBI

(En millones de S/.)

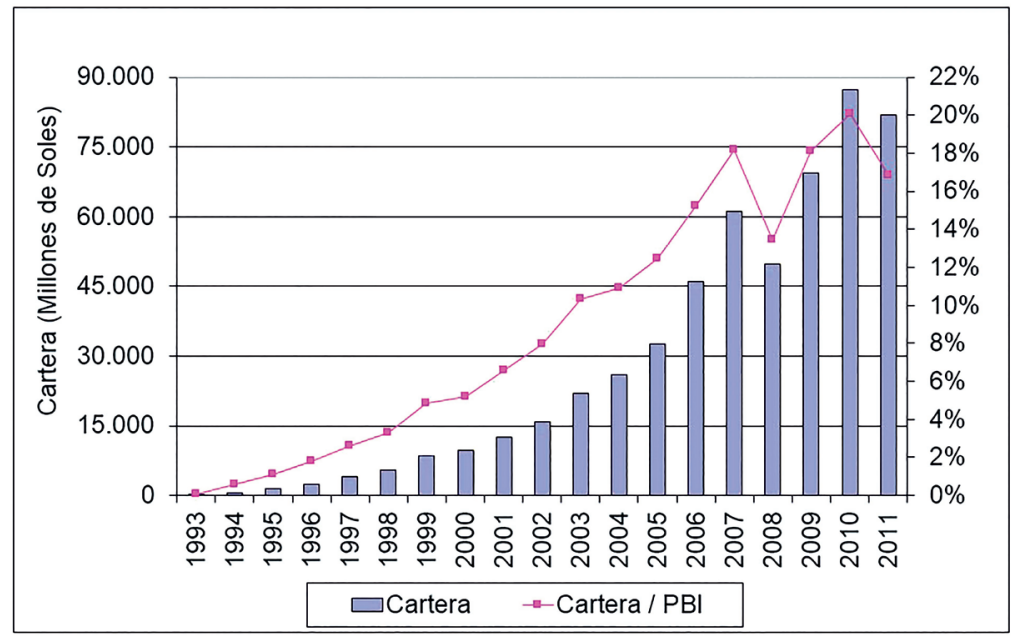

Fuente: SBS y AFP. Elaboración propia.

\section{TEORÍA DEL PORTAFOLIO}

Definido como el análisis cuantitativo de la administración óptima del riesgo para un conjunto de activos financieros. Como señalan Castillo y Lama $^{3}$ (2000): "Harry M. Markowitz al publicar su investigación dio origen a la Teoría de Portafolio modelar la racionalidad del inversionista en el mercado de capitales".

Esta teoría muestra la relación que debe de existir entre el riesgo y el retorno de un activo cualquiera. Para ello, se maximiza el rendimiento esperado a un cierto nivel de riesgo o se minimiza el riesgo a un nivel esperado de rendimiento.

En palabras de Elbaum ${ }^{4}$ : una organización de administración de inversiones reúne economistas, técnicos y otros expertos del mercado, quienes hacen proyecciones acerca de la economía y los mercados financieros, lo que se comunica a los analistas de valores. Los analistas hacen predicciones acerca de los valores de los que son responsables y se resumen en clasificaciones, información que se trasmite al comité de inversiones. Se considera lo siguiente:

1. Examinar las finanzas, la política, la economía $\mathrm{y}$ las condiciones sociales actuales y futuras. El inversionista debe enfocarse en las condiciones esperadas de corto y mediano plazo para utilizarlos en la construcción del portafolio.

2. Declaración de la política. El inversionista necesita definir cuáles son sus necesidades de corto y de largo plazo, la familiaridad con el mercado y las expectativas.

3 Castillo P. y Lama R. 2000. Evaluación de portafolio de inversionistas institucionales: fondos mutuos y fondos de pensiones. BCRP. Documentos de trabajo.

4 Elbaum M. 2004. Administración de carteras de inversión. Machi Grupo Editores. Buenos Aires. 
3. Implementación del plan a través de la construcción del portafolio. El inversionista debe de enfocarse en conocer sus riesgos en un nivel mínimo.

Continuando el razonamiento de Elbaum(2004):

El objetivo central de la Administración de Carteras es la construcción de un Portafolio. Para lograr esto, un administrador de inversiones no debe perder de vista:

1. Definir qué clase de activos van a ser incluidos en el portafolio

2. Perfil de riesgo del inversor

3. Principios de Diversificación

4. Longitud del plazo de inversión, y si es uno o varios períodos

5. Definición del índice de referencia o "benchmark" del portafolio.

6. Asignación estratégica de cada clase de activo en el largo plazo

7. Asignación táctica de cada clase de activos en el corto plazo.

8. Estrategia de selección a usar dentro de cada clase de activo.

\section{EL MODELO DE VALUACIÓN DE ACTIVOS DE CAPITAL (CAPM)}

La teoría CAPM es una herramienta comúnmente usada para medir el riesgo de los activos. Esta teoría se basa en la premisa de que los retornos de un activo o portafolio dependen solamente del riesgo sistemático o de mercado. El modelo plantea una relación lineal entre los retornos y el riesgo ${ }^{5}$.

$$
E\left(R_{j}\right)=R_{f}+\beta_{j}\left[E\left(R_{M}\right)-R_{f}\right]
$$

Donde: $\mathrm{E}(\mathrm{Rj})$ es la tasa de retorno esperada sobre un activo o portafolio particular, $\mathrm{Rf}$ es la tasa libre de riesgo; j representa el riesgo asumido por el activo o "portafolio j" y se define como la covarianza entre los retornos del activo riesgoso " $j$ " y los del portafolio del mercado " $m$ ", dividido por la varianza del portafolio del mercado; $\mathrm{E}(\mathrm{RM})$ es el retorno del portafolio de mercado esperado.

\section{Figura No 03:}

Frontera eficiente en presencia de títulos libres de riesgo

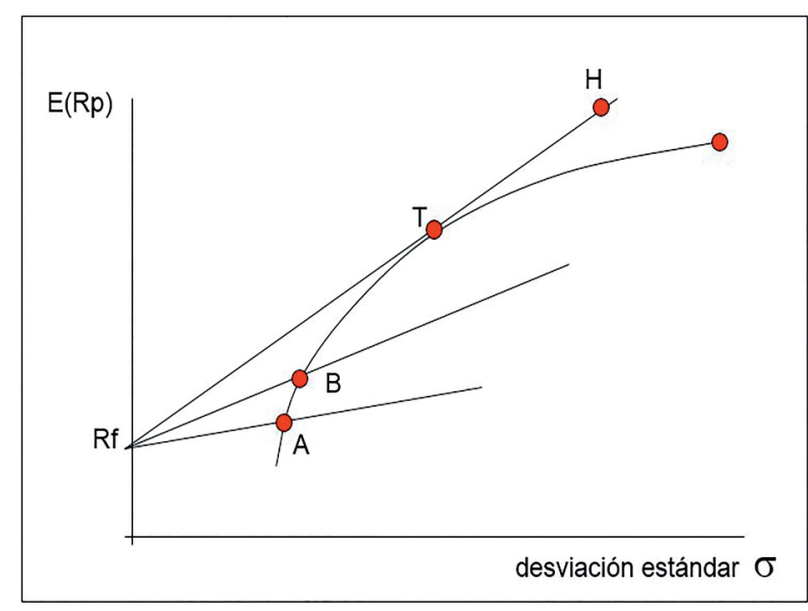

Fuente: Maria B Collatti. “Teoría de Carteras”

\section{INDICADORES DE GESTIÓN}

\section{Indicador de Sharpe}

William F. Sharpe ${ }^{6}$ en 1966 propone un cociente que viene determinado por la pendiente de la recta que conecta la cartera sin riesgo a la cartera riesgosa. Representa la prima de riesgo unitaria para el inversor. En otras palabras, proporciona el nivel de rendimiento por unidad de riesgo:

$$
\mathrm{Sp}=(\mathrm{Rp}-\mathrm{Rf}) / \sigma \mathrm{p}
$$

Donde $S p$ es el cociente de Sharpe de la cartera riesgosa $\mathrm{P}, \mathrm{Rp}$ es el rendimiento de la cartera riesgosa $\mathrm{P}$, $\mathrm{Rf}$ es la tasa sin riesgo y op es la volatilidad (medida de riesgo) de la cartera riesgosa $\mathrm{P}$.

\section{Indicador de Treynor}

El cociente de $\operatorname{Treynor}^{7}(\mathrm{Tp})$, propuesto en 1965 por Jack Treynor, viene determinado por la siguiente expresión:

$$
\mathrm{Tp}=(\mathrm{Rp}-\mathrm{Rf}) / \beta \mathrm{p}
$$

Donde Tp es el rendimiento del portafolio por unidad de riesgo, $\mathrm{Rf}$ es el rendimiento de un activo de libre de riesgo, $\mathrm{Rp}$ es el rendimiento del portafolio evaluado y $\beta$ p es el parámetro del modelo CAPM.

5 Ross S. 1999. Finanzas corporativas. $5^{\circ}$ edición. Ed McGrawHill. México.

6 Serge Zancanella. 1999. Les fonds de placement: mesures de la performance sur le marché Suisse. Université de Genève. Genève.

7 Ídem (8). 


\section{Indicador de Jensen}

Esta medida propuesta por Michael C. Jensen ${ }^{8}$ en 1968 se basa también en el riesgo sistemático y en el Modelo de Valuación de Activos de Capital (CAPM). A partir de estos resultados, Jensen determina la prima de riesgo para un nivel de riesgo sistemático dado, a la cual puede pretender todo fondo de colocación que practica una política de buy and hold. Va del principio que si el gestor de cartera anticipó correctamente los precios de equilibrio del mercado y que se propone diversificar su cartera de manera eficiente en el sentido de Markowitz, la cartera debe entonces situarse sobre la LMA:

El índice de Jensen es precisamente el valor obtenido para alfa:

$$
\alpha=(\mathrm{Rp}-\mathrm{RF})-\beta \mathrm{p}(\mathrm{Rm}-\mathrm{RF})+\mathrm{et}
$$

Donde $\alpha$ es el índice de Jensen, $\mathrm{Rp}$ es el rendimiento del portafolio; RF el rendimiento del activo libre de riesgo; $\mathrm{Rm}$ es el rendimiento del mercado, $\beta$ es la sensibilidad del portafolio a las fluctuaciones en el mercado de valores; $y$ et es el término de error que se comporta como ruido blanco.

\section{ANÁLISIS DE LA INFORMACIÓN}

En la presente investigación, con el propósito de evaluar el desempeño del portafolio de inversiones de las AFP's peruanas bajo el periodo de crisis financiera 2007 - 2011, se trabajó según diferentes niveles de análisis y desde distintos puntos de vista financieros se evaluó dicho desempeño. Los hallazgos y resultados se presentan a continuación.

\section{Análisis según modelo CAPM}

Aplicando el modelo CAPM a la información obtenida de la SBS y AFP, se ha construido la Tabla $\mathrm{N}^{\circ} 01$, que tiene como propósito determinar técnicamente la tasa de rendimiento apropiada del fondo tipo 2 para cada AFP. Asimismo previamente hemos obtenido la beta para cada AFP, que es la sensibilidad del activo al riesgo sistemático.

Tabla No 01:

Modelo de Valuación de Activos de Capital CAPM

\begin{tabular}{|c|c|c|c|c|c|c|c|c|}
\hline \multirow{2}{*}{\multicolumn{2}{|c|}{ Horizonte }} & \multirow[b]{2}{*}{ Integra } & \multicolumn{3}{|c|}{$E\left(R_{i}\right)=R_{f}+\beta .\left(R_{m}-R_{f}\right)$} & & \multicolumn{2}{|c|}{$\beta_{i}=\frac{\sigma_{i, m}}{\sigma_{m}^{2}}$} \\
\hline & & & Prima & Profuturo & SPP & IGBVL & CDBCRP & \\
\hline $\begin{array}{l}\mathrm{Ri}_{\text {CAPM }} \\
E(r)\end{array}$ & $\begin{array}{r}3.8249 \\
10.9724\end{array}$ & $\begin{array}{r}3.8427 \\
11.6945\end{array}$ & $\begin{array}{r}3.8478 \\
12.7253\end{array}$ & $\begin{array}{r}3.8403 \\
11.8616\end{array}$ & $\begin{array}{r}3.8368 \\
11.7663\end{array}$ & $\begin{array}{l}4.4267 \\
4.4267\end{array}$ & 3.6596 & 0.7670 \\
\hline$\sigma_{\mathrm{i}}$ & 20.3440 & 19.8658 & 20.4397 & 19.9798 & 20.1207 & 11.6793 & 0.1800 & 11.6798 \\
\hline$\rho_{\mathrm{i}, \mathrm{m}}$ & 0.1237 & 0.1403 & 0.1402 & 0.1377 & 0.1340 & 1.0000 & 0.0488 & \\
\hline$\sigma_{\mathrm{i}, \mathrm{m}}$ & 29.3845 & 32.5620 & 33.4653 & 32.1298 & 31.5006 & 136.4072 & 0.1026 & \\
\hline$\beta_{\mathrm{i}}$ & 0.2154 & 0.2387 & 0.2453 & 0.2355 & 0.2309 & 1.0000 & 0.0008 & \\
\hline
\end{tabular}

Fuente: Elaboración propia.

\section{Análisis según el modelo de portafolio}

Siguiendo la metodología de la moderna teoría de portafolio de Markowitz con el propósito de determinar teóricamente el portafolio óptimo (es decir, la mejor combinación de activos), se trabajó la formación de portafolios para cada una de las AFP, que se muestra en la figura $\mathrm{N}^{\circ} 04$. 
Figura No 04:

Portafolios óptimos para las AFP's
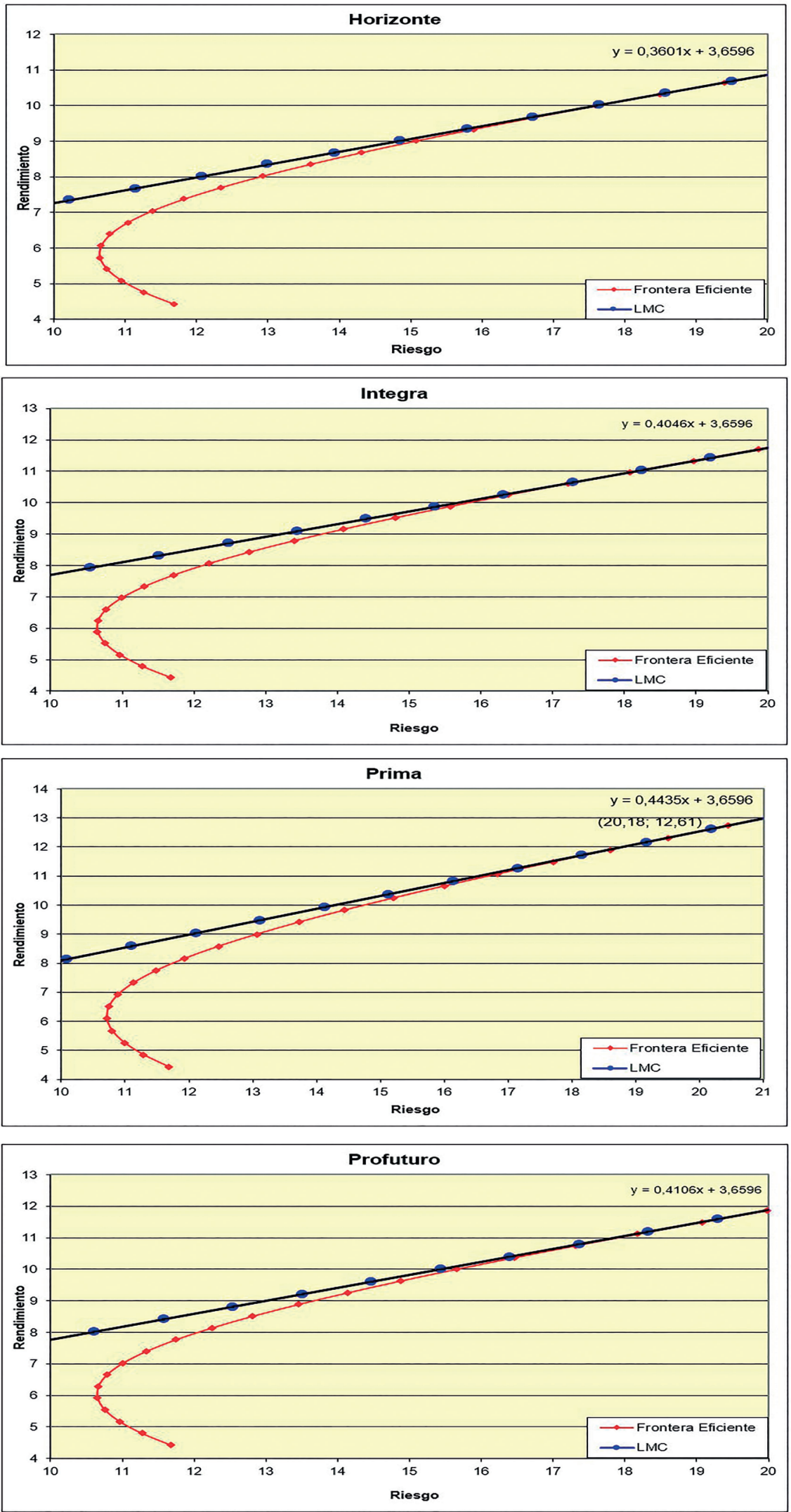

Fuente: Elaboración propia.

104/ QVIPURAMAVOC | Vol. 24(45 A) 2016 


\section{Análisis de Desempeño}

Tabla No 02:

Mercado privado de pensiones en el Perú

\begin{tabular}{|l|r|r|r|r|}
\hline \multicolumn{5}{|c|}{ Periodo : Enero 2006 - Diciembre 2011 } \\
\hline AFP & $\begin{array}{c}\text { Media } \\
\text { Esperada }\end{array}$ & $\begin{array}{c}\text { Desviacion } \\
\text { Estandar }\end{array}$ & Maximo & \multicolumn{1}{c|}{ Minimo } \\
\hline Horizonte & 10,9724 & 20,3440 & 50,5149 & $-32,0414$ \\
Integra & 11,6945 & 19,8658 & 50,1655 & $-31,1068$ \\
Prima & 12,7390 & 20,4422 & 53,2692 & $-31,3995$ \\
Profuturo & 11,8616 & 19,9798 & 51,6194 & $-31,3681$ \\
IGBVL & 4,4267 & 11,6793 & 38,4598 & $-37,2797$ \\
CDBCRP & 3,6596 & 0,1800 & 4,4318 & 1,3600 \\
\hline
\end{tabular}

Medida de Gestión de las AFP's

\begin{tabular}{|l|r|c|r|r|r|r|}
\hline \multicolumn{7}{|c|}{ Periodo: Enero 2006 - Diciembre 2011 } \\
\hline \multicolumn{1}{|c|}{ AFP } & INDICE & PENDIENTE & DIFERENCIA & \multicolumn{1}{c|}{ INDICE } & PENDIENTE & DIFERENCIA \\
\hline & SHARPE & LMC & & TREYNOR & LMA & \\
\hline Horizonte & 0,3595 & 0,06568 & 0,2938 & 27,3020 & 0,76705 & 26,5349 \\
\hline Integra & 0,4045 & & 0,3388 & 27,9531 & & 27,1861 \\
\hline Prima & 0,4441 & & 0,3785 & 30,5162 & & 29,7491 \\
\hline Profuturo & 0,4105 & & 0,3448 & 28,3307 & & 27,5636 \\
\hline
\end{tabular}

Medida de Selectividad de los Portafolio Manager de las AFP's

\begin{tabular}{|l|c|c|c|c|}
\hline \multicolumn{5}{|c|}{ Indice de Jensen : rp - rf $=\alpha p+\beta(\mathrm{rm}-\mathrm{rf})+$ et } \\
\hline \multicolumn{5}{|c|}{ Periodo: Enero 2006 - Diciembre 2011} \\
\hline AFP & $\alpha \mathrm{p}$ & \multicolumn{1}{|c|}{$\alpha \mathrm{p}$} & $\beta$ & $\beta$ \\
\hline & SELECTNIDAD & test $-\mathrm{t}$ & SENSIBILIDAD & test - t \\
\hline Horizonte & 7,1073 & 2,9211 & 0,2678 & 1,2796 \\
\hline Integra & 7,8144 & 3,2985 & 0,2874 & 1,4103 \\
\hline Prima & 8,8511 & 3,6290 & 0,2975 & 1,4180 \\
\hline Profuturo & 7,9799 & 3,3440 & 0,2895 & 1,4102 \\
\hline
\end{tabular}

Medida de Market Timing de los Portafolio Manager de las AFP's

\begin{tabular}{|c|c|c|c|c|c|c|}
\hline \multicolumn{7}{|c|}{ Indice de Treynor-Mazuy: $r p-r f=\alpha p+\beta 1(r m-r f)+\beta 2(r m-r f) 2+$ et } \\
\hline \multicolumn{7}{|c|}{ Periodo: Enero 2006 - Diciembre 2011} \\
\hline AFP & $a p$ & ap & $\beta 1$ & $\beta 1$ & $\beta 2$ & $\beta 2$ \\
\hline & SELECTNIDAD & test - $t$ & SENSIBILIDAD & test $-t$ & TIMING & test $-t$ \\
\hline Horizonte & 11,0688 & 4,3855 & 0,2593 & 1,3350 & $-0,0293$ & $-3,5093$ \\
\hline Integra & 11,6289 & 4,7226 & 0,2792 & 1,4735 & $-0,0282$ & $-3,4637$ \\
\hline Prima & 12,8281 & 5,0716 & 0,2889 & 1,4844 & $-0,0294$ & $-3,5154$ \\
\hline Profuturo & 11,8651 & 4,7930 & 0,2811 & 1,4757 & $-0,0287$ & $-3,5092$ \\
\hline
\end{tabular}

Fuente: Elaboración propia. 
En el desarrollo gráfico del análisis de portafolio óptimo para cada AFP observamos el par ordenado (riesgo, rentabilidad) sugieren que el SPP, en comparación al mercado bursátil peruano, obtiene rentabilidades menores, mientras que los riesgos asumidos son muy altos, lo cual es corroborado en el análisis CAPM anterior donde se observa $\sigma$ (el riesgo) y $\mathrm{E}(\mathrm{r})$ (la rentabilidad). Luego, esta situación también es corroborada en la posterior Tabla $\mathrm{N}^{\circ} 02$ de análisis de desempeño, donde los rendimientos hallados son poco significativos sobre la línea de mercado de capitales.

En el análisis de desempeño de la Tabla $\mathrm{N}^{\circ} 02$, se evalúa a cada AFP's, de acuerdo a su gestión de portafolio. Para ese propósito se procedió a aplicar:

Índice de Sharpe: Mide los rendimientos de la prima de riesgo por unidad de riesgo en una estrategia de inversión. Este indicador se confrontará con la línea de mercado de capitales.

Índice de Treynor: Mide los rendimientos de una inversión que no tiene riego diversificable por unidad de riesgo de mercado asumido. Este indicador se confrontará con la línea de mercado de activos.

Medida de Jensen: Determina la rentabilidad anormal de un portafolio sobre el retorno teórico esperado. Esta medida analiza la habilidad de Selectividad del administrador, es decir, la capacidad para elegir aquellos activos que el mercado ha sub valuado o sobre valuado.

Medida de Treynor-Mazuy: Esta medida analiza la habilidad de Market Timing de los administradores de portafolio, es decir, la capacidad para anticiparse sistemáticamente a los movimientos del mercado, con lo que se busca, conseguir mayores retornos, o menores pérdidas.

\section{CONCLUSIONES}

1. En el periodo agudo de crisis (2008-2009) los fondos de pensiones disminuyeron en $50 \%$ o más, lo cual implicaría que los administradores de portafolio de las AFPs asumieron riesgos incluso por encima de los riesgos asumidos por los participantes del mercado bursátil peruano.
2. La evaluación de desempeño de gestión, de los administradores de portafolio de las AFPs, medida por los cuatro indicadores estudiados, muestran la ausencia de un comportamiento competitivo.

\section{REFERENCIAS BIBLIOGRÁFICAS}

1. ALEXANDER, G.; SHARPE, W. \& BAILEY, J. (2003). Fundamentos de Inversiones. $3^{\circ} \mathrm{Ed}$. México.

2. BREALEY, R. \& MYERS, S. (2006). Principios de Finanzas Corporativas. $8^{\circ}$ Ed. Mc Graw Hill. Madrid.

3. CASTILLO, P. \& LAMA, R. (1998). Evaluación de portafolio de los inversionistas institucionales: fondos mutuos y fondos de pensiones, BCRP, Documentos de Trabajo. Lima.

4. ELBAUM, M. (2004). Administración de carteras de inversión. Machi Grupo Editores. Buenos Aires.

5. MORÓN, E. \& CARRANZA, E. (2003). Diez años del Sistema Privado de Pensiones: avances, retos y reformas. Ed. Universidad del Pacifico. Lima.

6. NELSON, D. (1991). "Conditional Hetoroskedasticity in Assest Returns: a New Approach”. Econometrica. Vol. 59. No2.

7. ROOS, S.; WESTERFIELD, R. \& JAFFE, J. (1999). Finanzas Corporativas. 5 Ed. Mc Graw Hill. México.

8. SÁNCHEZ,D. (2012). "Portafolio de inversiones del SPP peruano y su desempeño durante las crisis 2007 - 2011”. Tesis. UNMSM. Lima.

9. TITMAN, S. (1993). "Performance Measurment without Benchmarks: an Examination of mutual Fund Returns". Journal of Bussines. Vol. 66 No1.

10. ZANCANELLA, S. (1999). "Les fonds de placement: mesures de la performance sur le marché Suisse”. Université de Genève. Genéve.

11. BBC News UK. (2009). Financial Crisis: How it happened. BBC. London.

12. Superintendencia de Bancos, Seguros y AFP (2005 - 2012). Boletines. Lima. 